УДК 517.925.4

\author{
M. M. SHEREMETA
}

\title{
PSEUDOSTARLIKE AND PSEUDOCONVEX DIRICHLET SERIES OF ORDER $\alpha$ AND TYPE $\beta$
}

M. M. Sheremeta. Pseudostarlike and pseudoconvex Dirichlet series of order $\alpha$ and type $\beta$, Mat. Stud. 54 (2020), 23-31.

The concepts of the pseudostarlikeness of order $\alpha \in[0,1)$ and type $\beta \in(0,1]$ and the pseudoconvexity of order $\alpha$ and type $\beta$ are introduced for Dirichlet series with null abscissa of absolute convergence. In terms of coefficients, the pseudostarlikeness and the pseudoconvexity criteria of order $\alpha$ and type $\beta$ are proved. Let $h \geq 1, \Lambda=\left(\lambda_{k}\right)$ be an increasing to $+\infty$ sequence of positive numbers $\left(\lambda_{1}>h\right.$. We call a conformal function of the form $F(s)=$ $e^{s h}+\sum_{k=1}^{\infty} f_{k} \exp \left\{s \lambda_{k}\right\}, s=\sigma+i t$, in $\Pi_{0}=\{s: \operatorname{Re} s<0\}$ pseudostarlike of order $\alpha \in[0,1)$ and type $\beta \in(0,1]$ if

$$
\left|\frac{F^{\prime}(s)}{F(s)}-h\right|<\beta\left|\frac{F^{\prime}(s)}{F(s)}-(2 \alpha-h)\right|, \quad s \in \Pi_{0} .
$$

The main results of the article are contained in Theorems 1 and 2 . Theorem 1 states: If $\alpha \in[0,1)$ and $\beta \in(0,1]$ such that

$$
\sum_{k=1}^{\infty}\left\{(1+\beta) \lambda_{k}-2 \beta \alpha-h(1-\beta)\right\}\left|f_{k}\right| \leq 2 \beta(h-\alpha)
$$

then the function $F$ is pseudostarlike of order $\alpha$ and type $\beta$. The corresponding results for Hadamard compositions of such series are also established.

1. Introduction. Let $S$ be the class of analytic functions

$$
f(z)=z+\sum_{n=2}^{\infty} f_{n} z^{n}
$$

univalent in $\mathbb{D}=\{z:|z|<1\}$. Function $f \in S$ is said to be starlike if $f(\mathbb{D})$ is starlike domain concerning of the origin. It is well known [1, p. 202] that the condition $\operatorname{Re}\left\{z f^{\prime}(z) / f(z)\right\}>$ $0(z \in \mathbb{D})$ is necessary and sufficient for the starlikeness of $f$. A. W. Goodman ([2], see also $\left[3\right.$, p. 9]) proved that if $\sum_{n=2}^{\infty} n\left|f_{n}\right| \leq 1$ then function (1) is starlike. The concept of the starlikeness of function (1) got the series of generalizations. I.S. Jack ([4]) studied starlike functions of order $\alpha \in[0,1)$, i. e. such functions (1), for which $\operatorname{Re}\left\{z f^{\prime}(z) / f(z)\right\}>\alpha(z \in \mathbb{D})$. It is proved [4], [3, p. 13] that if $\sum_{n=2}^{\infty}(n-\alpha)\left|f_{n}\right| \leq 1-\alpha$ then function (1) is starlike function

2010 Mathematics Subject Classification: 30B50, 30D45.

Keywords: Dirichlet series; pseudostarlikeness; pseudoconvexity; Hadamard composition. doi:10.30970/ms.54.1.23-31

(C) M. M. Sheremeta, 2020 
of order $\alpha$. A function $f$ of the form (1) is called starlike function of order $\alpha \in[0,1)$ and type $\beta \in(0,1]$ when

$$
\left|\frac{z f^{\prime}(z)}{f(z)}-1\right|<\beta \cdot\left|\frac{z f^{\prime}(z)}{f(z)}+1-2 \alpha\right|
$$

This concept was introduced by V. P. Gupta ([5]).

We point out that the concept of $p$-valent starlike function $f(z)=z^{p}+\sum_{n=p+1}^{\infty} f_{n} z^{n}$ has appeared comparatively recently (see, for example, [6], [7] and [3, p. 14]).

Let $\Sigma$ be the class of functions

$$
f(z)=\frac{1}{z}+\sum_{n=1}^{\infty} f_{n} z^{n}
$$

analytic in $\mathbb{D}_{0}=\{z: 0<|z|<1\}$. The function $f \in \Sigma$ is said to be meromorphic starlike of order $\alpha \in[0,1)$ if $\operatorname{Re}\left\{-z f^{\prime}(z) / f(z)\right\}>\alpha\left(z \in \mathbb{D}_{0}\right)$. O.P. Juneja and T.R. Reddy [8] proved (see also [3, p. 14]) that if $\sum_{n=2}^{\infty}(n+\alpha)\left|f_{n}\right| \leq 1-\alpha$ then function (2) is meromorphic starlike function of order $\alpha$. According to B. A. Uralegaddi ([9]) the function (2) is said meromorphic starlike function of order $\beta \in(0,1]$ if $\left|z f^{\prime}(z)+f(z)\right|<\beta\left|z f^{\prime}(z)-f(z)\right|$ for all $z \in \mathbb{D}_{0}$. Finally, combining these definitions, M. L. Mogra, T. R. Reddy and O. P. Juneja ([10]) call a function $f \in \Sigma$ to be meromorphic starlike of order $\alpha \in[0,1)$ and type $\beta \in(0,1]$ if

$$
\left|z f^{\prime}(z)+f(z)\right|<\beta\left|z f^{\prime}(z)+(2 \alpha-1) f(z)\right|, \quad z \in \mathbb{D}_{0},
$$

and prove that if

$$
\sum_{n=1}^{\infty}((1+\beta) n+\beta(2 \alpha-1)+1)\left|f_{n}\right| \leq 2 \beta(1-\alpha),
$$

then the function (2) is meromorphic starlike of order $\alpha$ and type $\beta$. Using this statement, O. M. Mulyava and Yu. S. Trukhan ([11]) indicated conditions on the parameters $a_{1}^{(1)}, a_{2}^{(1)}$, $a^{(0)}, a_{2}^{(0)}, a_{3}^{(0)}$ of the differential equation of S. Shah $z^{2} w^{\prime \prime}+\left(a_{1}^{(1)} z^{2}+a_{2}^{(1)} z\right) w^{\prime}+$ $+\left(a_{1}^{(0)} z^{2}+a_{2}^{(0)} z+a_{3}^{(0)}\right) w=0$, under which this equation has a meromorphic starlike solution of order $\alpha$ and type $\beta$.

For power series $f_{j}(z)=\sum_{k=0}^{\infty} f_{k, j} z^{k}(j=1,2)$ the series $\left(f_{1} * f_{2}\right)(z)=\sum_{k=0}^{\infty} f_{k, 1} f_{k, 2} z^{k}$ is called the Hadamard composition (product) [12,13]. Properties of this composition obtained by J. Hadamard found applications $([13,14])$ in the theory of the analytic continuation of the functions represented by power series. We remark also that singular points of the Hadamard composition are investigated in the article [15].

L. Zalzman [16] studied Hadamard compositions of univalent functions (1). For the functions $f_{j}(z)=1 / z+\sum_{k=1}^{\infty} f_{k, j} z^{k} \in \Sigma(j=1,2)$ M. L. Mogra ([17]) defined Hadamard composition as $\left(f_{1} * f_{2}\right)(z)=1 / z+\sum_{k=1}^{\infty} f_{k, 1} f_{k, 2} z^{k}$ and proved, for example, that if the functions $f_{j}$ are meromorphically starlike of order $\alpha_{j} \in[0,1)$ and $f_{k, j} \geq 0$ for all $k \geq 1$ then $f_{1} * f_{2}$ is meromorphically starlike of order $\alpha=\max \left\{\alpha_{1}, \alpha_{2}\right\}$. Hadamard compositions of functions from the classes $S$ and $\Sigma$ were studied also by J. H. Choi, Y. C. Kim and S. Owa ([18]), M. K. Aouf and H. Silverman ([19]), J. Liu and R. Srivastava ([20]) and many other mathematicians.

Since Dirichlet series with positive increasing to $+\infty$ exponents are direct generalizations of power series, here was a necessity of a construction of the geometrical theory for the class of Dirichlet series, absolutely convergent in the half-plane $\Pi_{0}=\{s: \operatorname{Re} s<0\}$. The paper [21] is devoted to solve this problem (see also [3, p. 135-154]). 
So, let $h \geq 1, \Lambda=\left(\lambda_{k}\right)$ be an increasing to $+\infty$ sequence of positive numbers $\left(\lambda_{1}>h\right)$ and $S D(\Lambda, 0)$ be the class of Dirichlet series

$$
F(s)=e^{s h}+\sum_{k=1}^{\infty} f_{k} \exp \left\{s \lambda_{k}\right\}, \quad s=\sigma+i t,
$$

with the exponents $\Lambda$ and the abscissa of absolute convergence $\sigma_{a}[F]=0$. It is known [21] that each function $F \in S D(\Lambda, 0)$ is non-univalent in $\Pi_{0}$, but there exist conformal in $\Pi_{0}$ functions (3), and if $\sum_{k=2}^{\infty} \lambda_{k}\left|f_{k}\right| \leq \lambda_{1}$ then function (3) is conformal in $\Pi_{0}$. A conformal function (3) in $\Pi_{0}$ is said to be pseudostarlike if $\operatorname{Re}\left\{F^{\prime}(s) / F(s)\right\}>0$ for $s \in \Pi_{0}$. In [21] (see also [3, p. 139]) it is proved that if $\sum_{k=2}^{\infty} \lambda_{k}\left|f_{k}\right| \leq \lambda_{1}$ then function (3) is pseudostarlike.

The proposed paper continues the study of the geometric properties of Dirichlet series that are absolutely convergent in the half-plane.

2. Pseudostarlikeness of functions from $S D(\Lambda, 0)$. A conformal function $(3)$ in $\Pi_{0}$ is said to be pseudostarlike of order $\alpha$ if

$$
\operatorname{Re}\left\{F^{\prime}(s) / F(s)\right\}>\alpha \in[0,1), \quad s \in \Pi_{0} .
$$

Since the inequality $|w-h|<|w-(2 \alpha-h)|$ holds if and only if $\operatorname{Re} w>\alpha$, function (3) is pseudostarlike of order $\alpha$ if and only if

$$
\left|\frac{F^{\prime}(s)}{F(s)}-h\right|<\left|\frac{F^{\prime}(s)}{F(s)}-(2 \alpha-h)\right|, \quad s \in \Pi_{0} .
$$

In view of (5) we call conformal function (3) in $\Pi_{0}$ pseudostarlike of order $\alpha \in[0,1)$ and type $\beta \in(0,1]$ if

$$
\left|\frac{F^{\prime}(s)}{F(s)}-h\right|<\beta\left|\frac{F^{\prime}(s)}{F(s)}-(2 \alpha-h)\right|, \quad s \in \Pi_{0} .
$$

Theorem 1. Let $F \in S D(\Lambda, 0)$ be a function of the form (3). If $\alpha \in[0,1)$ and $\beta \in(0,1]$ such that

$$
\sum_{k=1}^{\infty}\left\{(1+\beta) \lambda_{k}-2 \beta \alpha-h(1-\beta)\right\}\left|f_{k}\right| \leq 2 \beta(h-\alpha)
$$

then the function $F$ is pseudostarlike of order $\alpha$ and type $\beta$.

Proof. Clearly, (6) holds if and only if

$$
\left|F^{\prime}(s)-h F(s)\right|-\beta\left|F^{\prime}(s)-(2 \alpha-h) F(s)\right|<0, \quad s \in \Pi_{0} .
$$

On the other hand,

$$
\begin{gathered}
\left|F^{\prime}(s)-h F(s)\right|-\beta\left|F^{\prime}(s)-(2 \alpha-h) F(s)\right|= \\
=\left|h e^{s h}+\sum_{k=1}^{\infty} \lambda_{k} f_{k} \exp \left\{s \lambda_{k}\right\}-h e^{s h}-h \sum_{k=1}^{\infty} f_{k} \exp \left\{s \lambda_{k}\right\}\right|- \\
-\beta\left|h e^{s h}+\sum_{k=1}^{\infty} \lambda_{k} f_{k} \exp \left\{s \lambda_{k}\right\}-(2 \alpha-h) e^{s h}-(2 \alpha-h) \sum_{k=1}^{\infty} f_{k} \exp \left\{s \lambda_{k}\right\}\right|=
\end{gathered}
$$




$$
=\left|\sum_{k=1}^{\infty}\left(\lambda_{k}-h\right) f_{k} \exp \left\{s \lambda_{k}\right\}\right|-\beta\left|2(h-\alpha) e^{s h}+\sum_{k=1}^{\infty}\left(\lambda_{k}-2 \alpha+h\right) f_{k} \exp \left\{s \lambda_{k}\right\}\right| .
$$

Since $-|a+b| \leq-|a|+|b|$ and $\sigma<0$, in view of (7) we get

$$
\begin{gathered}
\left|F^{\prime}(s)-h F(s)\right|-\beta\left|F^{\prime}(s)-(2 \alpha-h) F(s)\right| \leq \\
\leq\left|\sum_{k=1}^{\infty}\left(\lambda_{k}-h\right) f_{k} \exp \left\{s \lambda_{k}\right\}\right|-\left|2 \beta(h-\alpha) e^{s h}\right|+\left|\beta \sum_{k=1}^{\infty}\left(\lambda_{k}-2 \alpha+h\right) f_{k} \exp \left\{s \lambda_{k}\right\}\right| \leq \\
\leq \sum_{k=1}^{\infty}\left(\lambda_{k}-h\right)\left|f_{k}\right| \exp \left\{\sigma \lambda_{k}\right\}-2 \beta(h-\alpha) e^{\sigma h}+\beta \sum_{k=1}^{\infty}\left(\lambda_{k}-2 \alpha+h\right)\left|f_{k}\right| \exp \left\{\sigma \lambda_{k}\right\}= \\
=e^{\sigma h}\left(\sum_{k=1}^{\infty}\left\{(1+\beta) \lambda_{k}-2 \beta \alpha+h(1-\beta)\right\}\left|f_{k}\right| \exp \left\{\sigma\left(\lambda_{k}-h\right)\right\}-2 \beta(h-\alpha)\right)< \\
<\sum_{k=1}^{\infty}\left\{(1+\beta) \lambda_{k}-2 \beta \alpha-h(1-\beta)\right\}\left|f_{k}\right|-2 \beta(h-\alpha) \leq 0,
\end{gathered}
$$

i. e. (8) holds.

Theorem 2. If a function $F \in S D(\Lambda, 0)$ of form (3) is pseudostarlike of order $\alpha \in[0,1)$ and type $\beta \in(0,1]$ and $f_{k} \leq 0$ for all $k \geq 1$ then inequality ( 7$)$ holds.

Proof. Since function (3) is pseudostarlike of order $\alpha$ and type $\beta$ and $f_{k}=-\left|f_{k}\right|$ for all $k \geq 1$ in view of (8) as above we have for all $s \in \Pi_{0}$

$$
\left|\frac{-\sum_{k=1}^{\infty}\left(\lambda_{k}-h\right)\left|f_{k}\right| \exp \left\{s \lambda_{k}\right\}}{2(h-\alpha) e^{s h}-\sum_{k=1}^{\infty}\left(\lambda_{k}-2 \alpha+h\right)\left|f_{k}\right| \exp \left\{s \lambda_{k}\right\}}\right|=\left|\frac{F^{\prime}(s)-h F(s)}{F^{\prime}(s)-(2 \alpha-h) F(s)}\right|<\beta .
$$

Therefore,

$$
\operatorname{Re} \frac{\sum_{k=1}^{\infty}\left(\lambda_{k}-h\right)\left|f_{k}\right| \exp \left\{s \lambda_{k}\right\}}{2(h-\alpha) e^{s h}-\sum_{k=1}^{\infty}\left(\lambda_{k}-2 \alpha+h\right)\left|f_{k}\right| \exp \left\{s \lambda_{k}\right\}}<\beta,
$$

whence for all $\sigma<0$ we obtain

$$
\frac{\sum_{k=1}^{\infty}\left(\lambda_{k}-h\right)\left|f_{k}\right| \exp \left\{\sigma \lambda_{k}\right\}}{2(h-\alpha) e^{\sigma h}-\sum_{k=1}^{\infty}\left(\lambda_{k}-2 \alpha+h\right)\left|f_{k}\right| \exp \left\{\sigma \lambda_{k}\right\}}<\beta .
$$

Letting $\sigma \rightarrow 0$ from here we get

$$
\sum_{k=1}^{\infty}\left(\lambda_{k}-h\right)\left|f_{k}\right| \leq \beta\left(2(h-\alpha)-\sum_{k=1}^{\infty}\left(\lambda_{k}-2 \alpha+h\right)\left|f_{k}\right|\right)
$$

whence (7) follows. 
Choosing $\beta=1$ from Theorems 1 and 2 we obtain the following statement.

Corollary 1. In order for function (3) to be pseudostarlike of order $\alpha \in[0,1)$ it is sufficient and in the case when $f_{k} \leq 0$ for all $k \geq 1$ it is necessary that

$$
\sum_{k=1}^{\infty}\left(\lambda_{k}-\alpha\right)\left|f_{k}\right| \leq h-\alpha
$$

3. Pseudostarlikeness of functions from $\Sigma D(\Lambda, 0)$. Let $h \geq 1, \Lambda=\left(\lambda_{k}\right)$ be an increasing to $+\infty$ sequence of positive numbers and $\Sigma D(\Lambda, 0)$ be the class of Dirichlet series

$$
F(s)=e^{-s h}+\sum_{k=1}^{\infty} f_{k} \exp \left\{s \lambda_{k}\right\}, \quad s=\sigma+i t,
$$

absolutely convergent in $\Pi_{0}$. Dirichlet series (9) is called $\Sigma$-pseudostarlike of order $\alpha \in[0,1)$ if

$$
\operatorname{Re}\left\{F^{\prime}(s) / F(s)\right\}<-\alpha \in[0,1), \quad s \in \Pi_{0} .
$$

Since the inequality $|w+h|<|w+(2 \alpha-h)|$ holds if and only if $\operatorname{Re} w<-\alpha$, function (10) is $\Sigma$-pseudostarlike of order $\alpha$ if and only if

$$
\left|\frac{F^{\prime}(s)}{F(s)}+h\right|<\left|\frac{F^{\prime}(s)}{F(s)}+(2 \alpha-h)\right|, \quad s \in \Pi_{0} .
$$

In view of (11) we call function (10) $\Sigma$-pseudostarlike of order $\alpha \in[0,1)$ and type $\beta \in(0,1]$ if

$$
\left|\frac{F^{\prime}(s)}{F(s)}+h\right|<\beta\left|\frac{F^{\prime}(s)}{F(s)}+(2 \alpha-h)\right|, \quad s \in \Pi_{0} .
$$

Theorem 3. Let $F \in \Sigma D(\Lambda, 0)$ be a function of form (10). If $\alpha \in[0,1)$ and $\beta \in(0,1]$ such that

$$
\sum_{k=1}^{\infty}\left\{(1+\beta) \lambda_{k}+2 \beta \alpha+h(1-\beta)\right\}\left|f_{k}\right| \leq 2 \beta(h-\alpha)
$$

then $F$ is $\Sigma$-pseudostarlike of order $\alpha$ and type $\beta$.

Proof. Clearly, (13) holds if and only if

$$
\left|F^{\prime}(s)+h F(s)\right|-\beta\left|F^{\prime}(s)+(2 \alpha-h) F(s)\right|<0, \quad s \in \Pi_{0} .
$$

On the other hand, as in the proof of Theorem 1

$$
\begin{gathered}
\left|F^{\prime}(s)+h F(s)\right|-\beta\left|F^{\prime}(s)+(2 \alpha-h) F(s)\right|=e^{-\sigma h}\left(\left|\sum_{k=1}^{\infty}\left(\lambda_{k}+h\right) f_{k} \exp \left\{s\left(\lambda_{k}+h\right)\right\}\right|-\right. \\
\left.-\beta\left|2(\alpha-h)+\sum_{k=1}^{\infty}\left(\lambda_{k}+2 \alpha-h\right) f_{k} \exp \left\{s\left(\lambda_{k}+h\right)\right\}\right|\right) \leq \\
\leq e^{-\sigma h}\left(\sum_{k=1}^{\infty}\left(\lambda_{k}+h\right)\left|f_{k}\right| \exp \left\{\sigma\left(\lambda_{k}+h\right)\right\}-\right.
\end{gathered}
$$




$$
\begin{gathered}
\left.-\beta\left(2(h-\alpha)-\sum_{k=1}^{\infty}\left(\lambda_{k}+2 \alpha-h\right)\left|f_{k}\right| \exp \left\{\sigma\left(\lambda_{k}+h\right)\right\}\right)\right)= \\
=e^{-\sigma h}\left(\sum_{k=1}^{\infty}\left\{(1+\beta) \lambda_{k}+2 \beta \alpha+h(1-\beta)\right\}\left|f_{k}\right| \exp \left\{\sigma\left(\lambda_{k}+h\right)\right\}-2 \beta(h-\alpha)\right)< \\
\left.<e^{-\sigma h}\left(\sum_{k=1}^{\infty}\left\{(1+\beta) \lambda_{k}+2 \beta \alpha+h(1-\beta)\right\}\left|f_{k}\right|\right\}-2 \beta(h-\alpha)\right) \leq 0
\end{gathered}
$$

i.e. (15) holds.

Theorem 4. If the function $F \in \Sigma D(\Lambda, 0)$ of form (10) is $\Sigma$-pseudostarlike of order $\alpha$ and type $\beta$ and $f_{k} \geq 0$ for all $k \geq 1$ then inequality (14) holds.

Proof. Since function (10) is $\Sigma$-pseudostarlike of order $\alpha$ and type $\beta$ and $f_{k}=\left|f_{k}\right|$ for all $k \geq 1$ in view of (15) as above we have for all $s \in \Pi_{0}$

$$
\begin{gathered}
\operatorname{Re} \frac{\sum_{k=1}^{\infty}\left(\lambda_{k}+h\right) f_{k} \exp \left\{s \lambda_{k}\right\}}{2(h-\alpha) e^{-s h}-\sum_{k=1}^{\infty}\left(\lambda_{k}+2 \alpha-h\right) f_{k} \exp \left\{s \lambda_{k}\right\}} \leq \\
\leq \frac{\left|\sum_{k=1}^{\infty}\left(\lambda_{k}+h\right) f_{k} \exp \left\{s \lambda_{k}\right\}\right|}{\left|2(h-\alpha) e^{-s h}-\sum_{k=1}^{\infty}\left(\lambda_{k}+2 \alpha-h\right) f_{k} \exp \left\{s \lambda_{k}\right\}\right|}= \\
=\frac{\left|\sum_{k=1}^{\infty}\left(\lambda_{k}+h\right) f_{k} \exp \left\{s \lambda_{k}\right\}\right|}{\left|2(\alpha-h) e^{-s h}+\sum_{k=1}^{\infty}\left(\lambda_{k}+2 \alpha-h\right) f_{k} \exp \left\{s \lambda_{k}\right\}\right|}=\left|\frac{F^{\prime}(s)+h F(s)}{F^{\prime}(s)+(2 \alpha-h) F(s)}\right|<\beta,
\end{gathered}
$$

and, therefore, for all $\sigma<0$ we obtain

$$
\frac{\sum_{k=1}^{\infty}\left(\lambda_{k}+h\right) f_{k} \exp \left\{\sigma \lambda_{k}\right\}}{2(h-\alpha) e^{-\sigma h}-\sum_{k=1}^{\infty}\left(\lambda_{k}+2 \alpha-h\right) f_{k} \exp \left\{\sigma \lambda_{k}\right\}}<\beta .
$$

Letting $\sigma \rightarrow 0$ from here we get

$$
\sum_{k=1}^{\infty}\left(\lambda_{k}+h\right) f_{k} \leq \beta\left(2(h-\alpha)-\sum_{k=1}^{\infty}\left(\lambda_{k}+2 \alpha-h\right)\left|f_{k}\right|\right),
$$

whence (14) follows. Theorem 4 is proved.

Choosing $\beta=1$ from Theorems 3 and 4 we obtain the following statement.

Corollary 2. In order for function (10) to be $\Sigma$-pseudostarlike of order $\alpha \in[0,1)$ it is sufficient and in the case when $f_{k} \geq 0$ for all $k \geq 1$ it is necessary that

$$
\sum_{k=1}^{\infty}\left(\lambda_{k}+\alpha\right)\left|f_{k}\right| \leq h-\alpha
$$


4. Hadamard compositions. For Dirichlet series $F_{j}(s)=\sum_{k=0}^{\infty} f_{k, j} \exp \left\{s \lambda_{k}\right\}(j=1,2)$ in [22] a composition of Hadamard $\left(F_{1} * F_{2}\right)(s)=\sum_{k=0}^{\infty} f_{k, 1} f_{k, 2} \exp \left\{s \lambda_{k}\right\}$ is defined and some its properties are studied. Suppose that $F_{j} \in S D(\Lambda, 0)$. Then the Hadamard composition has the form

$$
\left(F_{1} * F_{2}\right)(s)=e^{s h}+\sum_{k=1}^{\infty} f_{k, 1} f_{k, 2} \exp \left\{s \lambda_{k}\right\}
$$

Corollary 1 implies the following statement.

Corollary 3. If the functions $F_{j} \in S D(\Lambda, 0)$ are pseudostarlike of orders $\alpha_{j} \in[0,1)$ and $f_{k, j} \leq 0$ for all $k \geq 1$ and $j=1,2$ then Hadamard composition $F_{1} * F_{2}$ is pseudostarlike of order $\alpha=\max \left\{\alpha_{j}: j=1,2\right\}$.

Indeed, from (9) it follows that $\left|f_{k, j}\right|<\left(h-\alpha_{j}\right) /\left(\lambda_{k}-\alpha_{j}\right)<1$ for all $k \geq 1$ and, therefore,

$$
\sum_{k=1}^{\infty} \frac{\lambda_{k}-\alpha_{1}}{h-\alpha_{1}}\left|f_{k, 1} f_{k, 2}\right| \leq \sum_{k=1}^{\infty} \frac{\lambda_{k}-\alpha_{1}}{h-\alpha_{1}}\left|f_{k, 1}\right|<1
$$

and similarly $\sum_{k=1}^{\infty} \frac{\lambda_{k}-\alpha_{2}}{h-\alpha_{2}}\left|f_{k, 1} f_{k, 2}\right|<1$, i. e.

$$
\sum_{k=1}^{\infty} \frac{\lambda_{k}-\max \left\{\alpha_{1}, \alpha_{2}\right\}}{h-\max \left\{\alpha_{1}, \alpha_{2}\right\}}\left|f_{k, 1} f_{k, 2}\right|=\sum_{k=1}^{\infty} \max \left\{\frac{\lambda_{k}-\alpha_{1}}{h-\alpha_{1}}, \frac{\lambda_{k}-\alpha_{2}}{h-\alpha_{2}}\right\}\left|f_{k, 1} f_{k, 2}\right|<1,
$$

and by Corollary 1 the function $F_{1} * F_{2}$ is pseudostarlike of order $\alpha=\max \left\{\alpha_{j}: j=1,2\right\}$.

Using Theorem 1 and 2 we get the following statement.

Corollary 4. Let the functions $F_{j} \in S D(\Lambda, 0)$ are pseudostarlike of orders $\alpha \in[0,1)$ and type $\beta_{j} \in(0,1]$ for $j=1,2$. If $f_{k, j} \leq 0$ for all $k \geq 1$ and $j=1,2$ then Hadamard composition $F_{1} * F_{2}$ is pseudostarlike of order $\alpha$ and type $\beta=\min \left\{\beta_{1}, \beta_{2}\right\}$.

Indeed, from (7) it follows that $\left|f_{k, j}\right|<\frac{2 \beta_{j} h-2 \beta_{j} \alpha}{\left(1+\beta_{j}\right) \lambda_{k}-2 \beta_{j} \alpha+h\left(1-\beta_{j}\right)}<1$ for all $k \geq 1$ and, therefore, as above we have

$$
\sum_{k=1}^{\infty} \frac{\left(1+\beta_{j}\right) \lambda_{k}-2 \beta_{j} \alpha-h\left(1-\beta_{j}\right)}{2 \beta_{j}(h-\alpha)}\left|f_{k, 1} f_{k, 2}\right| \leq 1, \quad j=1,2 .
$$

Hence

$$
\sum_{k=1}^{\infty} \max \left\{\frac{\left(1+\beta_{j}\right) \lambda_{k}-2 \beta_{j} \alpha-h\left(1-\beta_{j}\right)}{2 \beta_{j}(h-\alpha)}: j=1,2\right\}\left|f_{k, 1} f_{k, 2}\right| \leq 1 .
$$

Since

$$
\begin{gathered}
\max \left\{\frac{\left(1+\beta_{j}\right) \lambda_{k}-2 \beta_{j} \alpha-h\left(1-\beta_{j}\right)}{2 \beta_{j}(h-\alpha)}: j=1,2\right\}= \\
=\max \left\{\frac{\lambda_{k}-2 \alpha-h+\left(\lambda_{k}+h\right) / \beta_{j}}{2(h-\alpha)}: j=1,2\right\}=\frac{\lambda_{k}-2 \alpha-h+\left(\lambda_{k}+h\right) / \min \left\{\beta_{1}, \beta_{2}\right\}}{2(h-\alpha)},
\end{gathered}
$$

by Theorem 1 the function $F_{1} * F_{2}$ is pseudostarlike of order $\alpha$ and type $\beta=\min \left\{\beta_{1}, \beta_{2}\right\}$. 
Let us move on to the functions from the class $\Sigma D(\Lambda, 0)$. If $F_{j}(s)=e^{-h s}+\sum_{k=1}^{\infty} f_{k, j} \exp \left\{s \lambda_{k}\right\}$ $(j=1,2)$ then the Hadamard composition is defined by the equality

$$
\left(F_{1} * F_{2}\right)(s)=e^{-s h}+\sum_{k=1}^{\infty} f_{k, 1} f_{k, 2} \exp \left\{s \lambda_{k}\right\} .
$$

Corollary 2 implies the following statement.

Corollary 5. If the functions $F_{j} \in \Sigma D(\Lambda, 0)$ are $\Sigma$-pseudostarlike of orders $\alpha_{j} \in[0,1)$ and $f_{k, j} \geq 0$ for all $k \geq 1$ and $j=1,2$ then Hadamard composition $F_{1} * F_{2}$ is $\Sigma$-pseudostarlike of order $\alpha=\max \left\{\alpha_{j}: j=1,2\right\}$.

Indeed, from (16) it follows that $\left|f_{k, j}\right|<1$ for all $k \geq 1$ and, therefore, as above we have $\sum_{k=1}^{\infty} \frac{\lambda_{k}+\alpha_{1}}{h-\alpha_{1}}\left|f_{k, 1} f_{k, 2}\right|<1$ and $\sum_{k=1}^{\infty} \frac{\lambda_{k}+\alpha_{2}}{h-\alpha_{2}}\left|f_{k, 1} f_{k, 2}\right|<1$, i. e.

$$
\sum_{k=1}^{\infty} \frac{\lambda_{k}+\max \left\{\alpha_{1}, \alpha_{2}\right\}}{h-\max \left\{\alpha_{1}, \alpha_{2}\right\}}\left|f_{k, 1} f_{k, 2}\right|=\sum_{k=1}^{\infty} \max \left\{\frac{\lambda_{k}+\alpha_{1}}{h-\alpha_{1}}, \frac{\lambda_{k}+\alpha_{2}}{h-\alpha_{2}}\right\}\left|f_{k, 1} f_{k, 2}\right|<1,
$$

and by Corollary 2 the function $F_{1} * F_{2}$ is $\Sigma$-pseudostarlike of order $\alpha=\max \left\{\alpha_{j}: j=1,2\right\}$.

Using Theorems 3 and 4 and combining the proofs of Corollaries 4 and 5 , it is not difficult to prove the following statement.

Corollary 6. Let the functions $F_{j} \in \Sigma D(\Lambda, 0)$ are $\Sigma$-pseudostarlike of order $\alpha \in[0,1)$ and types $\beta_{j} \in(0,1]$. If $f_{k, j} \geq 0$ for all $k \geq 1$ and $j=1,2$ then Hadamard composition $F_{1} * F_{2}$ is $\Sigma$-pseudostarlike of order $\alpha$ and type $\beta=\min \left\{\beta_{1}, \beta_{2}\right\}$.

5. Pseudoconvex Dirichlet series. A conformal function (3) in $\Pi_{0}$ is said to be pseudoconvex if $\operatorname{Re}\left\{F^{\prime \prime}(s) / F^{\prime}(s)\right\}>0$ for $s \in \Pi_{0}$. In [21] and [3, p. 139] it is proved that if $\sum_{k=2}^{\infty} \lambda_{k}^{2}\left|f_{k}\right| \leq \lambda_{1}^{2}$ then function (3) is pseudoconvex. Here we call the function (3) pseudoconvex of order $\alpha \in[0,1)$ if $\operatorname{Re}\left\{F^{\prime \prime}(s) / F^{\prime}(s)\right\}>\alpha$, and pseudoconvex of order $\alpha$ and type $\beta \in(0,1]$ if

$$
\left|F^{\prime \prime}(s) / F^{\prime}(s)-h\right|<\beta\left|F^{\prime \prime}(s) / F^{\prime}(s)-(2 \alpha-h)\right|
$$

for all $s \in \Pi_{0}$.

Since $F^{\prime \prime}(s) / F^{\prime}(s)=G^{\prime}(s) / G(s)$, where $G(s)=e^{s h}+\sum_{k=1}^{\infty} g_{k} \exp \left\{s \lambda_{k}\right\}$ and $g_{k}=\lambda_{k} f_{k} / h$, the function $F$ is pseudoconvex of order $\alpha \in[0,1)$ and type $\beta \in(0,1]$ if and only if the function $G$ is pseudostarlike of order $\alpha \in[0,1)$ and type $\beta \in(0,1]$. Therefore, from the results proved above for pseudostarlike functions, one can easily obtain the corresponding results for pseudoconvex functions. For example, the following statement is valid.

Proposition 1. In order for the function $F \in S D(\Lambda, 0)$ of form (3) to be pseudoconvex of order $\alpha \in[0,1)$ and type $\beta \in(0,1]$ it is sufficient and in the case when $f_{k} \leq 0$ for all $k \geq 1$ it is necessary that $\sum_{k=1}^{\infty} \lambda_{k}\left\{(1+\beta) \lambda_{k}-2 \beta \alpha-h(1-\beta)\right\}\left|f_{k}\right| \leq 2 h \beta(h-\alpha)$.

Similarly, we call the function (10) $\Sigma$-pseudoconvex of order $\alpha \in[0,1)$ if $\operatorname{Re}\left\{F^{\prime \prime}(s) / F^{\prime}(s)\right\}<$ $-\alpha$, and $\Sigma$-pseudoconvex of order $\alpha$ and type $\beta \in(0,1]$ if

$$
\left|F^{\prime \prime}(s) / F^{\prime}(s)+h\right|<\beta\left|F^{\prime \prime}(s) / F^{\prime}(s)+(2 \alpha-h)\right|
$$

for all $s \in \Pi_{0}$. Now $F^{\prime \prime}(s) / F^{\prime}(s)=G^{\prime}(s) / G(s)$, where $G(s)=e^{-s h}+\sum_{k=1}^{\infty} g_{k} \exp \left\{s \lambda_{k}\right\}$ and $g_{k}=-\lambda_{k} f_{k} / h$. Since $g_{k} \geq 0$ if $f_{k} \leq 0$, from Theorem 3 and 4 we get the following statement.

Proposition 2. In order for the function $\Sigma F \in D(\Lambda, 0)$ of form (10) to be $\Sigma$-pseudoconvex of order $\alpha \in[0,1)$ and type $\beta \in(0,1]$ it is sufficient and in the case when $f_{k} \leq 0$ for all $k \geq 1$ it is necessary that $\sum_{k=1}^{\infty} \lambda_{k}\left\{(1+\beta) \lambda_{k}+2 \beta \alpha+h(1-\beta)\right\}\left|f_{k}\right| \leq 2 h \beta(h-\alpha)$. 


\section{REFERENCES}

1. Golusin G.M. Geometrical theory of functions of complex variables. - M.: Nauka, 1966. - 628 p. (in Russian); Engl. transl.: AMS: Translations of Mathematical monograph, 1969. - V.26. - 676 p.

2. Goodman A.W. Univalent functions and nonanalytic curves// Proc. Amer. Math. Soc. - 1957. - V.8, №3. - P. 597-601.

3. Sheremeta M.M. Geometric properties of analytic solutions of differential equations. - Lviv: Publisher I.E. Chyzhykov. -2019 . -164 p.

4. Jack I.S. Functions starlike and convex of order $\alpha / /$ J. London Math. Soc. - 1971. - V.3. - P. 469-474.

5. Gupta V.P. Convex class of starlike functions// Yokohama Math. J. - 1984. - V.32. - P. 55-59.

6. Owa S. On certain classes of p-valent functions with negative coefficients// Simon Stevin. - 1985. - V.59. - P. 385-402.

7. El-Ashwah R.M., Aouf M.K., Moustava A.O. Starlike and convexity properties for p-valent hypergeometric functions// Acta Math. Univ. Comenianae. - 2010. - V.79, №1. - P. 55-64.

8. Juneja O.P., Reddy T.R. Meromorphic starlike and univalent functions with positive coefficients// Ann. Univ. Mariae Curie-Sklodowska. - 1985. - V.39. - P. 65-76.

9. Uralegaddi B.A. Meromorphic starlike functions with positive coefficients// Kyungpook. Math. J. - 1989. - V.29, №1. - P. 64-68.

10. Mogra M.L., Reddy T.R., Juneja O.P. Meromorphic univalent functions with positive coefficients// Bull. Austral. Math. Soc. - 1985. - V.32, №2. - P. 161-176.

11. Truhan Yu.S., Mulyava O.M. On meromorphically starlike functions of the order $\alpha$ and the type $\beta$, which satisfy Shah's differential equations// Carpatian Math. Publ. - 2017. - V.9, №2. - P. 154-162.

12. Hadamard J. Théorème sur le series entières// Acta math. - 1899. - Bd.22. - S. 55-63.

13. Hadamard J. La série de Taylor et son prolongement analitique // Scientia phys.-math. - 1901. - №12. - P. 43-62.

14. Bieberbach L. Analytische Fortzetzung. - Berlin, 1955.

15. Korobeinik Yu.F., Mavrodi N.N. Singular points of the Hadamard composition// Ukr. Math. Journ.-1990. - V.42, №12. - P. 1711-1713. (in Russian); Engl. transl.: Ukr. Math. Journ. - 1990. - V.42, Issue 12. - P. $1545-1547$.

16. Zalzman L. Hadamard product of shlicht functions// Proc. Amer. Math. Soc. - 1968. - V.19, №3. P. 544-548.

17. Mogra M.L. Hadamard product of certain meromorphic univalent functions// J. Math. Anal. Appl. 1991. - V.157. - P. 10-16.

18. Choi J.H., Kim Y.C., Owa S. Generalizations of Hadamard products of functions with negative coefficients// J. Math. Anal. Appl. - 1996. - V.199. - P. 495-501.

19. Aouf M.K., Silverman H. Generalizations of Hadamard products of meromorphic univalent functions with positive coefficients// Demonstratio Mathematica. - 2008. - V.51, №2. - P. 381-388.

20. Liu J., Srivastava P. Hadamard products of certain classes of p-valent starlike functions// RACSM. 2019. - V.113. - P. 2001-205.

21. Holovata O.M., Mulyava O.M., Sheremeta M.M. Pseudostarlike, pseudoconvex and close-to-pseudoconvex Dirichlet series satisfying differential equations with exponential coefficients// Math. methods and physicomech. fields. - 2018. - V.61, №1. - P. 57-70. (in Ukrainian)

22. Mulyava O.M., Sheremeta M.M. Properties of Hadamard compositions of derivatives of Dirichlet series// Visnyk of Lviv Univ. Ser Mech. Math. - 2012. - Issue 77. - P. 157-166. (in Ukrainian)

Ivan Franko National University of Lviv m.m.sheremeta@gmail.com 